\title{
Criminologie
}

\section{Police et itinérance à Montréal : analyse des constats d'infraction aux règlements municipaux, 2005-2009}

\section{Rémi Boivin et Isabelle Billette}

Volume 45, numéro 2, automne 2012

URI : https://id.erudit.org/iderudit/1013727ar

DOI : https://doi.org/10.7202/1013727ar

Aller au sommaire du numéro

Éditeur(s)

Les Presses de l'Université de Montréal

ISSN

0316-0041 (imprimé)

1492-1367 (numérique)

Découvrir la revue

Citer cet article

Boivin, R. \& Billette, I. (2012). Police et itinérance à Montréal : analyse des constats d'infraction aux règlements municipaux, 2005-2009. Criminologie, 45(2), 249-276. https://doi.org/10.7202/1013727ar
Résumé de l'article

Les interventions policières à l'égard des personnes itinérantes font souvent l'objet de contestations sur la place publique, en particulier lorsqu'il y a émission de constats d'infraction en vertu des règlements municipaux. L'analyse présentée explore la surreprésentation de la population itinérante de Montréal parmi les personnes qui ont reçu au moins un constat d'infraction, pour la période 2005-2009. Les résultats indiquent que les personnes itinérantes reçoivent en moyenne trois fois plus de constats que la population non itinérante. Par contre, les trois quarts d'entre elles ne courraient pas le risque de recevoir un constat d'infraction au cours d'une année. Les résultats obtenus mettent en perspective la vision alarmiste souvent véhiculée par les médias. 


\title{
Police et itinérance à Montréal: analyse des constats d'infraction aux règlements municipaux, 2005-2009
}

\author{
Rémi Boivin \\ Professeur adjoint \\ École de criminologie, Université de Montréal \\ remi.boivin@umontreal.ca \\ Isabelle Billette \\ Conseillère en planification \\ Service de police de la ville de Montréal \\ isabelle.billette@spvm.qc.ca
}

RÉSUMÉ • Les interventions policières à l'égard des personnes itinérantes font souvent l'objet de contestations sur la place publique, en particulier lorsqu'il y a émission de constats d'infraction en vertu des règlements municipaux. L'analyse présentée explore la surreprésentation de la population itinérante de Montréal parmi les personnes qui ont reçu au moins un constat d'infraction, pour la période 2005-2009. Les résultats indiquent que les personnes itinérantes reçoivent en moyenne trois fois plus de constats que la population non itinérante. Par contre, les trois quarts d'entre elles ne courraient pas le risque de recevoir un constat d'infraction au cours d'une année. Les résultats obtenus mettent en perspective la vision alarmiste souvent véhiculée par les médias.

MOTS-CLÉS - Itinérance, police, intervention policière, règlements municipaux.

\section{Introduction $^{1}$}

Les interventions policières auprès des personnes itinérantes sont nombreuses. Chaque année, le Service de police de la Ville de Montréal

1. Nous souhaitons remercier Chloé Leclerc, Pierre Tremblay et les deux évaluateurs anonymes pour leurs commentaires et suggestions. Au Service de police de la Ville de Montréal, nous remercions Michelle Côté, de la Section recherche et planification, l'agente Any Gravel, du poste 21, et le directeur adjoint Denis Desroches qui ont lu et commenté l'article. 
(SPVM) doit répondre à plus de 10000 appels de service qui contiennent le mot «itinérant»-ou ses déclinaisons ${ }^{2}-$, des demandes auxquelles s'ajoutent les interventions effectuées par les policiers dans le cadre de leurs patrouilles. Les policiers interviennent de plusieurs façons: ils peuvent évincer ou faire circuler, recadrer verbalement, porter assistance, référer à des organismes de soutien, accompagner dans un centre hospitalier et sévir en remettant un constat d'infraction ou en arrêtant la personne lorsque la situation l'exige. Parmi celles-ci, les interventions qui mènent à l'émission d'un constat d'infraction sont souvent contestées. Certains auteurs indiquent que cette judiciarisation des personnes itinérantes est une forme de répression injustifiée et contraire à la volonté populaire (Bellot et al., 2005 ; Sylvestre, 2008; Raffestin, 2009). Le présent article examine un élément de l'hypothèse de la criminalisation de la population itinérante en analysant les constats d'infraction non criminelles émis en vertu de la réglementation municipale de Montréal entre 2005 et $2009^{3}$. L'analyse s'ajoute aux travaux qui remettent en question le bien-fondé de la stratégie d'émission de constats aux personnes itinérantes en offrant un aperçu quantitatif de la situation.

\section{Itinérance et sécurité urbaine}

\section{Le phénomène de l'itinérance}

L'ampleur du phénomène de l'itinérance est difficile à circonscrire de manière précise. La notion d'itinérance fait référence à un état souvent temporaire de personnes aux caractéristiques et aux trajectoires de vie très diversifiées qui vivent une situation de précarité, ce qui les garde à la marge des réseaux d'insertion traditionnels qui permettraient aux chercheurs de les joindre facilement (Rossi et al., 1987). S'ajoute à cela l'absence de consensus quant à la définition même du concept d'itinérance au-delà du fait qu'une personne est itinérante si elle n'a pas accès à un domicile fixe (voir notamment l'Institut canadien d'information

2. Un dénombrement manuel indique une moyenne de 35 appels par jour qui contiennent spécifiquement «itinérant(s)», «itinérante(s)» ou «itinérance» (35 appels X 365 jours = 12775 appels/année). Il s'agit d'une estimation conservatrice puisqu'elle n'inclut pas les cas où aucune référence n'est faite à la condition des personnes impliquées (ex.: « homme ivre couché dans la rue»), ni ceux où le libellé contient plutôt «sans-abri», "sdf», etc.

3. Les constats émis en vertu du Code de la sécurité routière sont exclus de l'analyse. 
sur la santé (ICIS), 2007; Roy et Hurtubise, 2008; Raffestin, 2009) ${ }^{4}$. En ce sens, l'épithète «itinérant» est utilisée ici comme un synonyme de «sans-abri» et de «sans domicile fixe».

Il est toutefois amplement documenté que plusieurs facteurs économiques, sociaux et individuels peuvent mener à un épisode d'itinérance (pour une revue des écrits, voir ICIS, 2007). Ainsi, en plus d'une pauvreté extrême, on note chez les personnes qui vivent en situation d'itinérance une forte prévalence de problèmes de santé mentale et physique, de dépendance à l'alcool et aux drogues, d'isolement, etc. (Rossi et al., 1987; Fournier, 2001 ; Roy et al., 2003 ; McGuire et Rosenbeck, 2004; Fazel et al., 2008; Roy et Hurtubise, 2008)5. En bref, les personnes itinérantes font souvent face à plusieurs problèmes concomitants qui renforcent leur vulnérabilité.

Malgré l'intérêt scientifique et politique porté à l'itinérance, relativement peu d'informations sont disponibles sur la taille et les caractéristiques précises de la population itinérante. Plusieurs méthodes sont utilisées pour l'estimation. Elles consistent généralement en un décompte des personnes qui utilisent les ressources communautaires (soupes populaires et centres d'hébergement) ou qui sont visibles durant la nuit. Le rapport de l'ICIS (2007: 10-11) regroupe les estimations disponibles pour une vingtaine de régions métropolitaines canadiennes, en précisant la méthode utilisée. Les estimations sont toutefois limitées par la méthodologie utilisée: par exemple, l'estimation pour Montréal ( $\mathrm{n}=1785$ personnes dans les refuges) est basée sur le dénombrement des places d'hébergement disponibles un jour donné et n'inclut pas les personnes qui utilisent rarement ou jamais les ressources. De façon plus importante, l'estimation tient pour acquis que l'offre répond à la demande, c'est-à-dire que toutes les personnes qui souhaitent être hébergées dans une ressource le peuvent. Pourtant, les organismes montréalais rapportent régulièrement fonctionner au-delà de leur capacité et refuser plusieurs demandes d'hébergement, faute d'espace $^{6}$. Ce type d'estimation produirait donc des chiffres très inférieurs à la réalité.

4. Cette définition très générale est elle-même problématique, puisqu'elle ne définit pas clairement la notion de «domicile fixe» (Rossi et al., 1987).

5. Quoique toujours élevée d'une étude à l'autre, la prévalence varie considérablement selon les auteurs.

6. Par exemple, «Les refuges de Montréal débordés» (Radio-Canada, 2010) et «Plus d'itinérants en raison de la pénurie de logements» (Guthrie, 2010). 
Fournier et al. (1998) ont mené un décompte systématique des utilisateurs des ressources communautaires de Montréal, sur une période d'un an. Leur étude démontre que 28214 personnes différentes ont utilisé les foyers d'hébergement, les soupes populaires et les centres de jour de Montréal, en 1996. Parmi ces personnes, au moins 12666 ont rapporté avoir été sans domicile fixe au cours des 12 mois précédents. Fournier et ses collègues ont aussi mené 135 entrevues qui leur ont permis de conclure qu' "environ $90 \%$ de cette population [itinérante] peut être rejointe dans les ressources communautaires [au cours d'une année]», pour une population totale d'un peu plus de 14000 individus (Fournier et al., 1998: 4). La Mission Old Brewery et le Secrétariat des partenariats de lutte contre l'itinérance estiment plutôt la population itinérante de Montréal à 30000 individus : cette estimation n'est toutefois pas basée sur une approche empirique comme celle de Fournier et al. (1998), de sorte qu'il est difficile d'en évaluer la validité. Ces résultats nous poussent à estimer que la population itinérante de Montréal est constituée de 14000 à 30000 individus.

\section{L'itinérance et les interventions policières}

L'itinérance interpelle la police de trois façons. Premièrement, les personnes itinérantes sont plus à risque de subir une victimisation criminelle que les personnes non itinérantes (Brassard et Cousineau, 2002). Par exemple, deux études états-uniennes ont démontré que le taux de victimisation des personnes itinérantes était de 3 à 5 fois plus élevé (Fitzpatrick et al., 1993; Lee et Schreck, 2005). En tant que groupe marginalisé, la population itinérante est plus à risque car elle est constituée de cibles intéressantes qui détiennent peu de moyens de protection (Gaetz, 2006). Les risques de victimisation sont particulièrement élevés pour les personnes itinérantes les plus vulnérables (ex.: santé physique fragile) (Fitzpatrick et al., 1993).

Deuxièmement, la présence de personnes itinérantes est une source d'insécurité pour plusieurs citoyens (Skogan, 1990; Markowitz et al., 2001). On les rend responsables de plusieurs nuisances (laisser traîner seringues et condoms, troubler la paix, flâner, uriner sur la voie publique, s'attrouper dans les parcs, etc.) généralement reliées à leurs conditions de vie et au fait qu'elles n'ont pas d'espace privé (Roy et Morin, 2007). Cette insécurité se traduit par un nombre important d'appels à la police concernant des personnes itinérantes. Aussi, suivant la thèse des «fenêtres 
brisées» (Broken windows, Wilson et Kelling, 1982), la gestion des incivilités est devenue une priorité des services de police depuis les années 1990. Récemment, Berk et MacDonald (2010) ont démontré qu'une politique de ce genre avait effectivement fait baisser la criminalité dans un secteur de Los Angeles (le Skid Row), réputé pour ses campements à ciel ouvert habités par des personnes itinérantes. En ce sens, le SPVM a adopté l'approche communautaire, qui vise à rapprocher la police de l'environnement de vie des citoyens. Depuis la fin des années 1990, les policiers sont encouragés à intervenir lorsque des personnes commettent des incivilités afin de diminuer leur occurrence, d'améliorer le sentiment de sécurité des citoyens et de diminuer la criminalité environnante.

Troisièmement, la proportion de personnes avec des antécédents criminels (avec ou sans incarcération) est plus élevée chez les itinérants que dans la population non itinérante (Snow et al., 1989; Metraux et Culhane, 2006). Les personnes itinérantes sont aussi surreprésentées parmi la population carcérale (Landreville et al., 1998; Robitaille et al., 2002; Greenberg et Rosenbeck, 2008). D'un côté, l'itinérance est présentée comme une situation criminogène en soi: les personnes commettent un plus grand nombre d'infractions lorsqu'elles deviennent itinérantes que lorsqu'elles ne le sont pas (McCarthy et Hagan, 1991). De l'autre côté, les crimes reprochés sont souvent des infractions de faible gravité ou des crimes contre les biens (Snow et al., 1989) et les personnes itinérantes qui ont des antécédents criminels ont des problèmes de santé physique et mentale plus sévères que les autres personnes itinérantes (McGuire et Rosenbeck, 2004).

\section{L'hypothèse de la criminalisation}

L'hypothèse de la criminalisation a d'abord été formulée pour l'intervention auprès des personnes souffrant de maladie mentale. Teplin (1984) a démontré que les suspects atteints de maladie mentale avaient plus de chances d'être arrêtés par la police que les autres. La criminalisation s'expliquerait par le manque de ressources dans la communauté à la suite de la désinstitutionnalisation des malades mentaux au cours des années 1970 et 1980, et par le manque de formation spécialisée des policiers (Morabito, 2007). Un manque de soutien adéquat aux personnes ayant des problèmes de santé mentale qui vivent dans la communauté augmente les probabilités que les policiers soient appelés pour intervenir (Pogrebin, 1986). 
L'hypothèse de la criminalisation implique que la police utilise de façon inappropriée la répression lors de l'intervention auprès de cette population. Elle est appuyée par les nombreux travaux qui soulignent la surreprésentation des individus souffrant de maladie mentale pris en charge par le système judiciaire. Par contre, la surreprésentation n'est pas suffisante pour arriver à la conclusion que la police offre un traitement différentiel à une population particulière. Engel et Silver (2001) ont révisé l'hypothèse de la criminalisation en tenant compte de l'impact de plusieurs facteurs: les caractéristiques individuelles du suspect (sexe, âge, origine culturelle), son attitude au moment de l'intervention policière (état d'ébriété, coopération, agressivité), le contexte de l'intervention (nombre d'observateurs, lieu, source de la plainte) et le cadre légal (preuves, possession d'une arme, gravité de l'infraction). Ils trouvent plutôt que les personnes qui souffrent de maladie mentale ont moins de chances d'être arrêtées, à circonstances égales (voir aussi Novak et Engel, 2005).

L'hypothèse de la criminalisation a ensuite été appliquée à la population itinérante (Landreville et al., 1998; National Law Center on Homelessness and Poverty (NLCHP), 2009). Sur le plan des pratiques policières, elle implique que les patrouilleurs interviendraient différemment auprès du groupe marginalisé, en favorisant notamment le recours à des pratiques répressives comme l'émission de constats d'infraction. Globalement, l'hypothèse implique plutôt que la population marginalisée recevrait un nombre anormalement élevé de constats d'infraction, et que cette surreprésentation serait due à la volonté de l'État de gérer le «problème» de l'itinérance en criminalisant ces personnes (Bellot $e t$ al., 2005). Cette hypothèse s'ajoute donc aux différents facteurs qui influencent le déroulement des interventions policières.

L'hypothèse de la criminalisation de la population itinérante est en fait une réaction en chaîne. L'état d'itinérance, en raison de l'absence d'espace privé, amène l'individu à recourir à des activités plus ou moins illicites (squeegee, quête, etc.) dans une logique de survie matérielle et sociale (pour se nourrir, se loger, parler, assouvir sa dépendance, etc.). Ces infractions sont parfois punies par l'émission d'un constat d'infraction. Puisque l'itinérance implique généralement une grande pauvreté, l'individu n'arrive pas à payer l'amende et risque d'être condamné à l'incarcération pour défaut de paiement ${ }^{7}$. En plus des préjudices que

7. Depuis plusieurs années, la Ville de Montréal a suspendu la délivrance de mandats d'emprisonnement pour non-paiement d'amendes sur son territoire. Les personnes itiné- 
cela implique pour la personne elle-même (emprisonnement, antécédents judiciaires), l'incarcération des personnes itinérantes a des coûts assumés par les citoyens. C'est ce qui amène Raffestin (2009) à parler d'«injustice programmée», puisqu'elle implique plusieurs acteurs qui se partagent la responsabilité de la situation. La criminalisation des infractions commises par des personnes itinérantes est la conséquence occasionnelle de l'intervention policière initiale (Landreville et al., 1998).

\section{Problématique}

Bellot et ses collègues (Bellot et al., 2005; voir aussi Raffestin, 2009) ont exploré de manière quantitative l'hypothèse de la criminalisation ${ }^{8}$ de la population itinérante de Montréal. Dans un premier temps, elles notent que le nombre de constats d'infraction remis à des personnes itinérantes pour des infractions criminelles a considérablement augmenté à la fin des années 1990, passant de 1069 en 1994 à 4188 en 2003 (2005: 43), pour une population de moins de 30000 individus. Leur analyse des caractéristiques (âge et sexe) des personnes qui ont reçu ces constats les porte à conclure que «personne n'est épargné par la répression» (2005: 49). Dans un deuxième temps, les auteures indiquent que la majorité des infractions reprochées aux personnes itinérantes «porte sur une présence refusée dans l'espace public et la consommation d'alcool dans le domaine public» (2005: 54). Autrement dit, les auteures interprètent leurs résultats comme une preuve de la judiciarisation des populations itinérantes à Montréal (voir aussi Larouche, 2009; Sylvestre, 2010).

Cette conclusion nous paraît hâtive pour trois raisons. Premièrement, elle néglige les interactions police-personnes itinérantes qui ne se concluent pas par l'émission d'un constat d'infraction. Ces situations sont nombreuses: par exemple, le SPVM reçoit chaque année plus de 10000 appels qui concernent des personnes itinérantes, mais a donné moins de 4000 constats d'infraction par année à ces personnes, incluant

rantes ne courent plus le risque d'être emprisonnées à la suite d'un constat d'infraction aux règlements municipaux. Or, cette pratique n'a pas été accompagnée d'une modification de la loi et le moratoire pourrait donc être levé en toute légalité.

8. Il ne faut pas confondre judiciarisation et criminalisation. Selon le Larousse, le terme judiciarisation indique «une propension à privilégier le recours à l'intervention judiciaire». Le terme criminalisation renvoie plutôt à un "processus plus global qui représente la transition de la juridiction civile vers la juridiction pénale». 
ceux qui découlent des interventions en patrouillant. Par ailleurs, dans le récit des faits des constats rédigés, les policiers indiquent souvent connaitre le contrevenant ou l'avoir déjà averti par le passé pour un comportement similaire avant d'émettre le constat. Deuxièmement, les critiques tiennent pour acquis que l'ensemble des personnes itinérantes sont susceptibles de recevoir un constat d'infraction. Le filet pénal s'étendrait de façon uniforme et aveugle à la population itinérante. On peut en déduire que les personnes itinérantes qui ont reçu un constat d'infraction devraient être un échantillon assez représentatif de l'ensemble de la population itinérante, ce qui reste à démontrer. Troisièmement, Bellot et al. (2005), entre autres, analysent uniquement les constats reçus par des personnes itinérantes, ce qui ne permet pas de les comparer aux constats reçus par des personnes non itinérantes (absence de groupe contrôle).

L'analyse qui suit explore un des éléments de l'hypothèse de la criminalisation, soit la surreprésentation des personnes itinérantes parmi les contrevenants aux règlements municipaux à Montréal. D'abord, nous tentons de contribuer au débat en comparant les constats reçus par des personnes itinérantes à ceux reçus par des personnes non itinérantes. Ensuite, nous comparons certaines caractéristiques sociodémographiques des personnes qui utilisaient des ressources communautaires (Fournier et al., 1998) à celles des personnes itinérantes qui ont reçu au moins un constat d'infraction en 2008-2009. Puis, nous comparons les caractéristiques des personnes itinérantes qui ont reçu un seul constat d'infraction et celles qui en ont reçu plusieurs durant la même période (2008-2009). Finalement, nous estimons la taille de la population itinérante à risque de recevoir un ou plusieurs constats d'infraction non criminelle à Montréal. Nos résultats suggèrent que les personnes itinérantes ne sont pas toutes susceptibles de faire l'objet de criminalisation et que des constats d'infraction sont donnés par les policiers dans certaines circonstances spécifiques.

\section{Méthodologie}

Les données utilisées sont issues du Module d'information policière, qui contient l'ensemble des infractions, criminelles ou non, enregistrées par le SPVM, ainsi que certaines informations sur les personnes impliquées, pour la période de 2005 à 2009. Cette période a été choisie pour deux raisons. Premièrement, en 2004, la procédure d'enregistrement 
des constats a été modifiée, de sorte que les banques de données contiennent maintenant plusieurs informations qui n'étaient pas disponibles auparavant sur les constats d'infraction non criminelles. Deuxièmement, le SPVM a mis sur pied une unité policière dédiée uniquement à la sécurité dans le métro en 2007. Bellot et al. (2005) indiquent que les agents du métro donnaient un nombre important de constats d'infraction aux personnes itinérantes jusqu'à 2004. Il sera donc possible d'observer l'évolution de la répression des personnes itinérantes depuis l'implantation de l'unité métro.

\section{Les constats émis à des personnes itinérantes}

Lors de l'émission d'un constat d'infraction, les policiers recueillent des informations sur la personne accusée (le contrevenant). Outre son nom et des détails sur le contexte de l'infraction (heure, lieu, etc.), les policiers doivent indiquer l'adresse de son domicile. Lorsque la personne est itinérante, il est possible: 1) que le policier n'indique aucune adresse; 2) que le contrevenant donne l'adresse d'une ressource communautaire; ou 3) que le contrevenant donne une fausse adresse. Une adresse manquante ne signifie pas toujours que la personne est itinérante, puisque l'adresse de résidence n'est pas nécessaire à l'émission d'un constat d'infraction. Nous avons donc considéré qu'un constat avait été émis à une personne itinérante:

1. Lorsque le contrevenant avait indiqué l'adresse d'une ressource communautaire pour personnes itinérantes. Ce critère a permis de repérer 18769 constats émis de 2005 à 2009. La méthode est la même que celle utilisée par Bellot et al. $(2005)^{9}$;

2. Lorsque l'adresse de résidence était manquante et que le contrevenant avait indiqué au moins une fois l'adresse d'une ressource communautaire durant la même année. Ainsi, 4654 constats supplémentaires ont été intégrés aux analyses.

La stratégie de repérage des constats d'infraction indique qu'en moyenne, un peu plus de 4600 constats d'infraction ont été remis à des personnes

9. Bellot et ses collaborateurs (2005) ont effectué l'extraction à partir de la banque générale de la Cour municipale de Montréal (le «plumitif»), tandis que nous l'avons faite à partir d'une banque de données compilée par le SPVM. Techniquement, cette distinction ne devrait pas avoir d'impact sur le nombre de constats identifiés, puisque tous les constats émis par le SPVM sont envoyés à la Cour municipale. 
itinérantes chaque année, de 2005 à 2009. Cette estimation est légèrement supérieure aux chiffres compilés par Bellot et al. (2005) pour l'année 2003 ( $n=4188$ constats). La différence peut s'expliquer en partie par l'ajout des constats pour lesquels l'adresse était manquante (moyenne de 930 par année), mais surtout par le fait que nos données n'incluent pas les constats émis par les agents de surveillance de la Société de transport de Montréal qui travaillent dans le métro. Cette limite influence principalement les données de 2005 et 2006, puisque depuis la création de l'unité métro en 2007, plusieurs constats sont émis dans le métro par des agents du SPVM et apparaissent donc dans les données analysées.

Nous avons aussi constitué un échantillon aléatoire stratifié de 200 constats émis en 2008-2009 à Montréal, dont la moitié $(\mathrm{n}=100)$ a été émise à des personnes itinérantes. Au moment de la rédaction du présent article, les informations les plus récentes disponibles dataient des années 2008 et 2009. Nous en avons extrait quatre informations supplémentaires: 1) l'origine de l'intervention policière; 2) l'infraction reprochée; 3) le nombre de personnes qui accompagnaient le contrevenant au moment de l'infraction; et 4) le montant de l'amende.

L'origine de l'intervention policière indique si le constat découlait d'une plainte formulée par un citoyen ou d'une observation faite en patrouillant. L'origine de l'intervention n'est pas toujours indiquée dans le récit des faits (manquante pour 50 cas sur 200; 25\%). Les infractions reprochées ont été regroupées en six catégories: les infractions pouvaient concerner 1) la consommation d'alcool ou de drogues (ex. : flâner ivre, consommer sur le domaine public); 2) le fait de salir l'espace public (ex.: uriner, jeter des déchets sur le sol); 3) l'occupation inappropriée de l'espace public (ex.: se trouver dans un parc fermé); 4) le bruit excessif (ex.: crier sans raison, injurier); 5) le refus d'obéir à un agent de la paix (ex.: entraver la circulation de piétons ou d'automobiles, malgré les avertissements); et 6) d'autres infractions (ex.: avoir un couteau, promener un chien sans laisse). Finalement, le nombre de personnes a été recodé de façon dichotomique: le contrevenant était accompagné ou non au moment de l'infraction.

\section{Les caractéristiques individuelles}

À partir de la banque de données sur l'ensemble des constats d'infraction, nous avons créé une seconde banque contenant des informations sur les contrevenants. Une personne est considérée itinérante si elle a 
reçu au moins un constat d'infraction et qu'elle a alors donné l'adresse d'une ressource communautaire pour personnes itinérantes. Puisque l'itinérance fait généralement référence à un état temporaire, nous avons considéré chaque année de 2005 à 2009 séparément. Ainsi, une personne peut être considérée itinérante en 2008, mais pas en 2009.

Outre la condition d'itinérance, cette banque de données inclut le sexe, l'âge (au 31 décembre 2008) et l'origine culturelle de la personne (Blanc/non-Blanc $\left.{ }^{10}\right)$, ainsi que le nombre de constats reçus par année, et les lieux de fréquentation (centre-ville, métro). Nous considérons qu'une personne fréquente le centre-ville (ou le métro) ${ }^{11}$ si elle a reçu un constat d'infraction à cet endroit, en 2008-2009.

La seconde banque a été créée à l'aide d'une clé contenant les trois premières lettres du nom de famille, la première lettre du prénom et la date de naissance (année, mois, jour). Cette approche permet de réduire les biais liés aux erreurs d'orthographe du nom, mais ne permet pas d'éviter les erreurs volontaires ou non de date de naissance. Cette clé unique permet de retracer l'implication des personnes dans les infractions criminelles commises à Montréal, de 2005 à 2009. Autrement dit, nous avons vérifié si les personnes itinérantes qui ont reçu des constats d'infraction ont aussi été suspectées ou arrêtées en lien avec des infractions criminelles commises durant la même période. Il est probable que les personnes qui ont des antécédents criminels soient plus surveillées ou connues des policiers, ce qui peut influencer la probabilité qu'elles reçoivent un constat d'infraction ${ }^{12}$. Les résultats des analyses des caractéristiques individuelles sont présentés plus loin.

10. Cette distinction est évidemment très réductrice. Par contre, notre expérience de travail et des discussions avec des policiers nous ont démontré que les policiers de Montréal s'entendaient bien (accord inter-juges) lorsqu'ils qualifiaient une personne de blanche ou de caucasienne, mais que la distinction entre d'autres origines culturelles (ex.: Arabes, Sud-Asiatiques, Noirs, Latino-Américains, Asiatiques) était plus laborieuse. Autrement dit, face à une personne blanche, la plupart des policiers indiqueront correctement qu'elle est blanche. Par contre, face à une personne basanée, une proportion non négligeable de policiers indiqueront une mauvaise origine ethnique.

11. À Montréal, les événements criminels et les constats d'infraction sont classés selon le lieu où ils ont été commis. Ainsi, le centre-ville correspond aux postes de quartier 12, 20, 21 et 22, alors que le métro correspond au 50.

12. Le fait d'utiliser une variable continue plutôt que dichotomique ne change pas les résultats des modèles multivariés présentés au tableau 3. Il semble qu'à partir du moment où ils ont été arrêtés pour un crime, les individus deviennent connus des policiers, puisqu'ils ont un historique judiciaire. Il reste possible que la lourdeur des antécédents criminels influence le style d'intervention policière, mais les données ne permettent pas de tester cette hypothèse. 


\section{Résultats}

\section{Les constats d'infraction}

L'hypothèse de la criminalisation implique d'abord une intervention différenciée en fonction d'une caractéristique de la population visée, dans le cas présent, les personnes itinérantes. Cette intervention pourrait se traduire par l'émission de constats pour des infractions particulières. Le tableau 1 compare les constats d'infraction remis à des personnes itinérantes et non itinérantes. Les colonnes «Personnes itinérantes» et «Personnes non itinérantes» du tableau présentent les données descriptives pour chacun des groupes; par exemple, 50\% des constats remis à des personnes itinérantes portaient sur une infraction liée à la consommation d'alcool ou de drogues, contre $41 \%$ des constats remis à des personnes non itinérantes. De la même façon, le montant moyen de l'amende exigée était de 145 \$ pour les personnes itinérantes,

TAB LEA U 1

Caractéristiques des constats d'infraction, 2008-2009

\begin{tabular}{|c|c|c|c|c|}
\hline & & $\begin{array}{l}\text { Personnes } \\
\text { itinérantes }\end{array}$ & $\begin{array}{c}\text { Personnes } \\
\text { non } \\
\text { itinérantes }\end{array}$ & $\begin{array}{c}\text { Différence } \\
\text { significative? } \\
(p<0,05)\end{array}$ \\
\hline \multirow{11}{*}{$\begin{array}{l}\text { Échantillon } \\
(n=200)\end{array}$} & Types d'infraction (\%) & & & \\
\hline & $\begin{array}{l}\text { Liée à la consommation } \\
\text { de drogues ou d'alcool }\end{array}$ & $50 \%$ & $41 \%$ & Non $^{1}$ \\
\hline & Salir l'espace public & $12 \%$ & $11 \%$ & Non $^{1}$ \\
\hline & Occupation de l'espace urbain & $15 \%$ & $10 \%$ & Non $^{1}$ \\
\hline & Cris/injures & $9 \%$ & $17 \%$ & Non $^{1}$ \\
\hline & Refus d'obéir à un agent & $10 \%$ & $10 \%$ & Non $^{1}$ \\
\hline & Autres & $4 \%$ & $11 \%$ & Non $^{1}$ \\
\hline & TOTAL & $100 \%$ & $100 \%$ & - \\
\hline & Contrevenant seul $(0 / N)$ & $85 \%$ & $69 \%$ & $\mathrm{Oui}^{2}$ \\
\hline & $\begin{array}{l}\text { Constat remis en réponse à une } \\
\text { plainte }(0 / N)\end{array}$ & $41 \%$ & $34 \%$ & $\mathrm{Non}^{2}$ \\
\hline & Montant moyen de l'amende $(\$)$ & 145 & 134 & Non $^{3}$ \\
\hline \multirow{3}{*}{$\begin{array}{l}\text { Total } \\
(n=40698)\end{array}$} & Émis au centre-ville (\%) & $51 \%$ & $27 \%$ & Oui ${ }^{1}$ \\
\hline & Émis dans le métro (\%) & $34 \%$ & $10 \%$ & Oui ${ }^{1}$ \\
\hline & $\begin{array}{l}\text { Nombre de constats par personne } \\
(2008-2009)\end{array}$ & 4,2 & 1,3 & Oui ${ }^{3}$ \\
\hline
\end{tabular}

Tests: ${ }^{1}$ Différence de proportion

${ }^{2}$ Tableau croisé

${ }^{3}$ Différence de moyenne 
contre $134 \$$ pour les personnes non itinérantes. La colonne de droite indique s'il existe une différence significative entre les deux groupes. Le tableau intègre les résultats de trois types d'analyses bivariées: des différences de moyenne, des tableaux croisés et des différences de proportion. Si les deux premières sont couramment utilisées en sciences sociales, les différences de proportion sont moins connues. Il s'agit d'un test basé sur la statistique $z$ qui permet de déterminer si une proportion associée à l'échantillon A est comparable à la proportion associée à l'échantillon $\mathrm{B}$, même si les deux échantillons sont de taille différente (voir Newcombe, 1998). Dans tous les cas, nous avons indiqué que la différence était significative si la valeur $p$ était inférieure à 0,05 .

Plusieurs similitudes ressortent quant au type d'infraction reprochée, à l'origine de l'intervention policière et au montant de l'amende. Soulignons d'ailleurs que le montant de l'amende est étroitement lié à la nature de l'infraction et que les policiers doivent respecter un montant minimal prévu par le règlement. On note que $41 \%$ des constats émis à des personnes itinérantes (et $34 \%$ de ceux émis à d'autres personnes) découlent explicitement d'une plainte formulée par un citoyen. Dans plusieurs autres cas, les policiers ont indiqué spécifiquement que leur présence à un endroit s'expliquait par une accumulation de plaintes et de problèmes récents. Le nombre de constats émis varie aussi en fonction des saisons, pour les deux groupes. Le nombre de constats émis en hiver est beaucoup moins élevé qu'en été, de sorte que la distribution mensuelle des constats suit un cycle annuel (voir annexe 1).

Il aurait été étonnant que des infractions similaires génèrent des différences significatives. Ce qui varie, c'est le contexte dans lequel les personnes reçoivent des constats. Plus de la moitié (51\%) des constats des personnes itinérantes ont été émis au centre-ville, et environ le tiers (34\%) dans le métro (pour un total de $85 \%$ des constats). Ces proportions sont significativement plus élevées que pour les personnes non itinérantes (27\% au centre-ville, $10 \%$ dans le métro). Elles reflètent probablement la répartition géographique réelle des personnes itinérantes. Cette affirmation est appuyée par le fait que la très grande majorité des ressources communautaires pour personnes itinérantes est située au centre-ville ${ }^{13}$. La répartition temporelle est aussi différente

13. Il y a un problème de simultanéité entre l'offre de services et la répartition géographique de la population itinérante. Pourquoi y a-t-il plus de personnes itinérantes au centre-ville? En partie parce qu'il s'y trouve des ressources, mais aussi parce que l'accès aux drogues y est plus facile, que les revenus potentiels de quête y sont plus élevés, que la 
(résultats non montrés); $45 \%$ des constats reçus par des personnes non itinérantes portent sur une infraction commise entre $23 \mathrm{~h} 00$ et $4 \mathrm{~h} 00$, heures de fréquentation des bars, entre autres. Les constats remis aux personnes itinérantes sont plus répartis durant la journée, et la majorité (70\%) sont donnés durant le jour, entre 10 h 00 et minuit $(0$ h 00). Enfin, les personnes itinérantes étaient plus susceptibles d'être seules au moment où elles ont reçu les constats d'infraction ( $85 \%$ vs $69 \%$ pour les personnes non itinérantes). Les personnes itinérantes semblent ainsi plus susceptibles de recevoir un constat lorsqu'elles sont plus visibles.

Une dernière différence significative vient du nombre de constats reçus par individu. Cette différence est probablement celle qui frappe le plus l'imaginaire des citoyens et elle est à l'origine de la plupart des critiques concernant l'intervention policière auprès des personnes itinérantes. En 2008-2009 (période de 24 mois), à Montréal, 33167 constats d'infraction à des règlements municipaux ont été donnés à 25630 personnes non itinérantes (moyenne de 0,65 constat par personne par année), alors que 7531 constats ont été remis à 1780 personnes itinérantes (moyenne de 2,12 par personne par année). Lorsqu'on fait le ratio entre le nombre de constats et la population totale, les personnes itinérantes sont énormément surreprésentées (environ 18 constats par 1000 habitants de Montréal vs 251-538 constats par 1000 personnes itinérantes).

Ces statistiques frappantes ne permettent toutefois pas de conclure que la police intervient de la même façon auprès de l'ensemble de la population itinérante (Engel et Silver, 2001). Elles supposent que les constats d'infraction sont distribués aléatoirement au sein de la population et que tous courent les mêmes risques d'en recevoir. La figure 1 présente la répartition du nombre de personnes itinérantes en fonction du nombre de constats d'infraction reçus en 2008-2009. Plus de la moitié $(54,2 \%)$ des personnes itinérantes qui ont reçu au moins un constat pendant ces 24 mois en ont reçu un seul, et $16,3 \%$ en ont reçu deux (moyenne d'un constat par année). Or, le nombre moyen de constats par personne pour la même période est de 4,3 (ligne pointillée). En plus, ces chiffres ne tiennent pas compte des personnes itinérantes qui n'ont pas reçu de constat durant la période. Il serait donc plus exact d'affirmer qu'un petit nombre de personnes itinérantes reçoit un grand nombre de constats, mais que la majorité en reçoit peu ou pas.

densité de personnes présentes offre un certain anonymat, etc. Pourquoi les ressources sont-elles situées à proximité du centre-ville? Parce que la population itinérante s'y trouve. C'est le cas classique de l'œuf et de la poule. 
F I G URE 1

Distribution du nombre de constats par personne itinérante, 2008-2009 (24 mois)

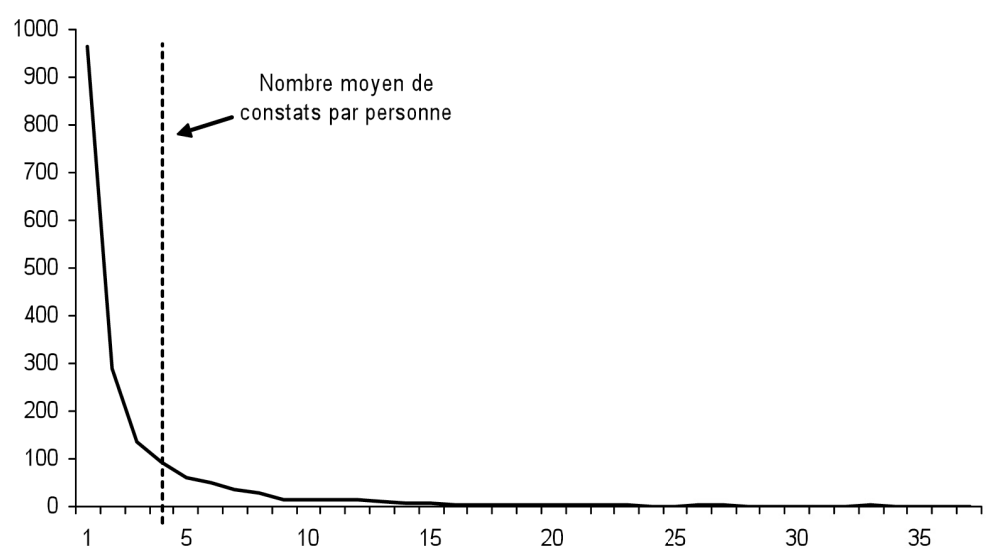

La judiciarisation des personnes itinérantes

Le fait que des personnes itinérantes reçoivent des constats d'infraction à Montréal est indéniable. Il est aussi indiscutable que le taux de constats par personne est significativement plus élevé pour les personnes itinérantes (voir tableau 1). Par ailleurs, l'hypothèse de la criminalisation implique que la police adopte une stratégie unique face aux personnes itinérantes, même si cette stratégie comporte des inconvénients manifestes. Dans le cas qui nous intéresse, cette stratégie devrait se traduire par l'émission de constats d'infraction à un échantillon assez représentatif de la population itinérante: autrement dit les personnes itinérantes qui reçoivent un ou plusieurs constats d'infraction devraient partager les mêmes caractéristiques sociodémographiques que celles qui n'en reçoivent pas. De la même façon, les personnes qui reçoivent un seul constat devraient partager les caractéristiques de celles qui en reçoivent plusieurs. Par extension, l'ensemble de la population itinérante devrait être à risque de recevoir au moins un constat d'infraction. 
Caractéristiques des personnes qui ont reçu des constats d'infraction

Les personnes itinérantes qui reçoivent un constat d'infraction représentent une partie de l'ensemble de la population itinérante: en 2008-2009, 1780 personnes itinérantes ont reçu un constat, alors que les estimations de la population itinérante oscillaient entre 14000 et 30000 personnes, soit une proportion entre 5,9\% à 12,7\%. Le tableau 2 compare les caractéristiques (sexe et âge) des 28214 utilisateurs des ressources communautaires recensés par Fournier et al. (1998) et des 1780 personnes itinérantes qui ont reçu un constat d'infraction en 2008-2009. L'approche est considérablement limitée par le fait qu'elle se base sur un recensement effectué une dizaine d'années plus tôt (1996). Les chiffres avancés par Fournier et al. (1998) n'ont jamais été mis à jour.

TABLEA U 2

Comparaison des caractéristiques des personnes itinérantes qui utilisaient les ressources communautaires et des personnes itinérantes qui ont reçu des constats d'infraction en 2008-2009 ${ }^{14}$

\begin{tabular}{|c|c|c|c|}
\hline & $\begin{array}{c}\text { Personnes } \\
\text { itinérantes } \\
\text { (Fournier } \\
\text { et al., 1998) }\end{array}$ & $\begin{array}{c}\text { Personnes } \\
\text { itinérantes } \\
\text { qui ont reçu un } \\
\text { constat d'infraction } \\
(2008-2009)\end{array}$ & $\begin{array}{c}\text { Différence } \\
\text { significative? }\end{array}$ \\
\hline Nombre de personnes & $28214^{1}$ & 1780 & - \\
\hline Sexe (\% hommes) & $77 \%$ & $92 \%$ & Oui ${ }^{* *}$ \\
\hline \multicolumn{4}{|l|}{ Âge } \\
\hline $18-29$ ans (\%) & $29 \%$ & $27 \%$ & Oui** \\
\hline $30-44$ ans (\%) & $36 \%$ & $34 \%$ & Non \\
\hline $45-64$ ans $(\%)$ & $26 \%$ & $36 \%$ & Oui ${ }^{* *}$ \\
\hline 65 ans et plus (\%) & $5 \%$ & $2 \%$ & Oui $i^{* *}$ \\
\hline
\end{tabular}

${ }^{*} \mathrm{p}<0,05{ }^{* *} \mathrm{p}<0,01$

1. Fournier et al. (1998) ont dénombré les clients des ressources communautaires (hébergement, soupes populaires et centres de jour). Parmi ces 28214 clients, 17000 avaient été sans domicile fixe à un moment durant leur vie et 12 666, au courant de la dernière année. Les caractéristiques de ces sous-groupes ne sont pas présentées. Nous tenons pour acquis qu'elles sont les mêmes.

14. Les personnes âgées de moins de 18 ans constituent environ $4 \%$ de la population itinérante (Fournier et al., 1998). L'intervention policière auprès des personnes mineures (de moins de 18 ans) est souvent différente de celle auprès d'une clientèle adulte. Par exemple, il est possible que les policiers préfèrent faire appel à d'autres intervenants (ex.: centres jeunesse), plutôt que d'émettre des constats d'infraction. Ces faits justifient l'exclusion des personnes de moins de 18 ans de l'analyse. 
Les personnes itinérantes qui ont reçu un constat d'infraction sont significativement différentes de l'ensemble de la population itinérante de Montréal. Les statistiques du tableau 2 indiquent que les hommes et les personnes de 45 à 64 ans sont surreprésentés parmi ceux qui ont reçu un constat. À l'inverse, les personnes de 18 à 29 ans et de plus de 65 ans sont sous-représentées.

Existe-t-il des différences parmi les personnes itinérantes qui reçoivent des constats d'infraction? En 2008-2009, un nombre similaire de personnes ont reçu un seul constat $(964 ; 54 \%)$ et deux constats ou plus $(816 ; 46 \%)$. Qu'est-ce qui les différencie? Le tableau 3 présente les résultats d'une analyse de régression logistique tentant de distinguer les personnes itinérantes qui ont reçu plusieurs constats d'infraction. Les coefficients présentés indiquent la probabilité d'être une de ces personnes. La valeur de référence est 1 . Une valeur inférieure à 1 signifie que la personne qui possède cette caractéristique a moins de chances de recevoir plusieurs constats d'infraction, alors qu'une valeur supérieure à 1 signifie qu'elle a plus de chances. Par exemple, une personne qui a commis une infraction criminelle au cours des cinq années à l'étude a 2,38 fois plus de chances d'avoir reçu au moins deux constats d'infraction en 2008-2009.

TABLEAU 3

Analyse de régression logistique visant à distinguer les personnes itinérantes qui ont reçu plus d'un constat d'infraction en 2008-2009

\begin{tabular}{|l|c|}
\hline & $\begin{array}{c}\text { A reçu plus d'un constat d'infraction } \\
\text { en 2008-2009 (Odds ratio) }\end{array}$ \\
\hline Nombre de cas inclus dans l'analyse & 813 \\
\hline Âge $^{1}$ & 1,00 \\
\hline Sexe (homme $=1$ ) & 0,62 \\
\hline Origine ethnique (non-Blanc = 1) & 0,87 \\
\hline $\begin{array}{l}\text { A commis une infraction criminelle à } \\
\text { Montréal entre 2005 et 2009 (oui }=1)\end{array}$ & $2,38^{* *}$ \\
\hline Fréquente le métro (oui = 1) & $2,51^{* *}$ \\
\hline $\begin{array}{l}\text { A connu une période d'itinérance entre 2005 } \\
\text { et 2007 (oui = 1) }\end{array}$ & $2,58^{* *}$ \\
\hline R carré (Nagelkerke) & $0,20^{* *}$ \\
\hline
\end{tabular}

$* * p<0,01$

1. Le fait de considérer l'âge selon les quatre catégories décrites au tableau 2 ne change pas les résultats obtenus. Aucune catégorie n'a un impact significatif sur la probabilité d'avoir reçu plus d'un constat d'infraction en 2008-2009. 
Les personnes itinérantes qui ont reçu deux constats ou plus sur une période de deux ans (2008-2009) ne se distinguent pas de celles qui en ont reçu un seul, sur le plan des caractéristiques sociodémographiques (sexe, âge, origine ethnique). Toutefois, les personnes qui ont aussi été arrêtées pour avoir commis une infraction criminelle avaient 2,38 fois plus de chances de recevoir plusieurs constats d'infraction. Ce résultat peut s'expliquer par trois mécanismes. Premièrement, les personnes qui ont commis un acte criminel sont probablement mieux connues des policiers de la ville. Elles sont surveillées plus étroitement, de sorte que leurs écarts de conduite sont plus visibles pour les policiers. Deuxièmement, lors d'une intervention, les policiers vérifient souvent l'identité des personnes impliquées. Les policiers sont possiblement plus sévères avec les personnes qui ont des antécédents criminels. Troisièmement, les personnes qui ont des antécédents criminels ont démontré leur potentiel délinquant. Il est possible qu'elles commettent simplement un plus grand nombre d'infractions, et qu'elles soient rattrapées par la loi des probabilités.

Les personnes qui fréquentent les installations du métro sont plus de deux fois $(2,51)$ plus susceptibles de recevoir de multiples constats. Il faut dire que le métro est un endroit fermé, où il est difficile de se cacher de la vue des passants. De plus, plus d'un demi-million de personnes utilisent le métro chaque jour pour se déplacer ${ }^{15}$, ce qui fait que les personnes itinérantes qui s'y trouvent sont très visibles. Enfin, la vocation particulière de cet espace (un lieu de transit) amène une gestion spécifique de son usage et un niveau de tolérance moins élevé de la part des agents.

Finalement, les personnes qui ont un long historique d'itinérance sont aussi plus susceptibles de recevoir de multiples constats. Celles qui avaient été itinérantes entre 2005 et 2007 avaient 2,58 fois plus de chances d'avoir reçu deux constats ou plus en 2008-2009. Ces individus ( $28 \%$ des personnes itinérantes) sont probablement plus connus des policiers de Montréal. Les personnes itinérantes chroniques sont aussi plus susceptibles de souffrir de troubles mentaux et d'avoir des problèmes de consommation d'alcool ou de drogues (Fournier, 2001; Fazel et al., 2008). D’ailleurs, parmi les 442 personnes itinérantes qui ont reçu deux constats ou plus en 2009 , presque la moitié $(n=209 ; 47 \%)$

15. Issu du rapport d'activités de la Société de transport de Montréal, (2008). 
ont reçu deux constats ou plus au cours d'au moins une autre année entre 2005 et 2008. La proportion est légèrement plus élevée pour les personnes qui ont reçu au moins trois constats en 2009 ( $n=151$; $54 \%$ ). Les proportions sont similaires pour toutes les années entre 2005 et 2008. Ainsi, certaines personnes itinérantes reçoivent un plus grand nombre de constats d'infraction, soit parce qu'elles sont plus ciblées par les interventions policières, soit parce qu'elles ont et conservent des comportements qui les exposent à de telles interventions policières. Les policiers n'étendraient donc pas uniformément - et aveuglément - le filet pénal.

Estimation des risques de recevoir un constat d'infraction

Parmi les personnes itinérantes qui reçoivent des constats d'infraction, la grande majorité ne reçoit qu'un seul constat d'infraction par année (les «1»), même si une certaine proportion en reçoit deux ou plus (tableau 4). De plus, il est raisonnable de penser qu'un nombre indéterminé de personnes itinérantes ne reçoivent pas de constat, même si leurs caractéristiques et/ou leurs comportements pourraient les exposer à une telle sanction. Il est nécessaire d'évaluer cette population (les «0») pour estimer les risques réels encourus par les personnes itinérantes de recevoir un constat. Si toutes les personnes itinérantes sont à risque, alors la somme des personnes qui ont reçu un constat et des personnes qui n'en ont pas reçu devrait se situer entre 14000 et 30000 . Si seulement une partie de la population itinérante est à risque, alors la somme ne devrait pas dépasser 14000 .

La méthode capture-recapture permet d'estimer la population à risque, à partir de la distribution des «1» et des « 2 ». Il s'agit d'une technique empruntée à la biologie animale, qui permet d'estimer les populations cachées ou difficiles à rejoindre. En criminologie, la méthode a été utilisée pour estimer les populations délinquantes de revendeurs de drogue (Bouchard et Tremblay, 2005; Charest, 2010), de planteurs de cannabis (Bouchard, 2007), de prostituées (Rossmo et Routledge, 1990; Roberts et Brewer, 2006) et de voleurs de voitures (Collins et Wilson, 1990). La technique a aussi été utilisée pour évaluer certains aspects des populations itinérantes (Fisher et al., 1994; Berry, 2007; van Hest et al., 2008). Elle repose sur l'idée que les personnes qui reçoivent un ou deux constats au cours d'une année partagent les mêmes caractéristiques que celles qui sont à risque mais n'en ont pas reçu, pour 
différentes raisons, dont le hasard ${ }^{16}$. Le tableau 4 présente les estimations de population dérivées de l'estimateur $Z$ de Zelterman (1988). Les estimations sont présentées pour chaque année, de 2005 à 2009. Avant 2007, plusieurs constats étaient émis par des agents de la sûreté du métro; nous avons ajusté la distribution des personnes itinérantes sur la base des données accumulées par Bellot et al. (2005). Ainsi, 385 personnes supplémentaires auraient reçu un seul constat d'infraction et 105 personnes, deux. Sans ces ajustements, la taille estimée de la population itinérante à risque est grandement sous-estimée, puisqu'elle néglige les constats émis par des agents du métro. Depuis 2007, la très grande majorité des constats dans le métro sont émis par des policiers du SPVM et sont donc comptabilisés dans les données utilisées.

TA B LEA U 4

Population itinérante à risque de recevoir un constat d'infraction

\begin{tabular}{|l|c|c|c|c|c|c|}
\hline & Population à risque & \multicolumn{4}{|c|}{ Population, selon le nombre de constats reçus } \\
\cline { 3 - 7 } Année & Estimateur de Zelterman & $0^{*}$ & 1 & 2 & $\begin{array}{l}3 \text { et } \\
\text { plus }\end{array}$ & $\begin{array}{l}\text { Total (au moins } \\
1 \text { constat) }\end{array}$ \\
\hline 2005 & 3749 & 2292 & 886 & 218 & 353 & 1457 \\
\hline 2006 & 3641 & 2129 & 917 & 246 & 349 & 1512 \\
\hline 2007 & 3301 & 2049 & 776 & 185 & 291 & 1252 \\
\hline 2008 & 2175 & 1081 & 598 & 209 & 287 & 1094 \\
\hline 2009 & 2068 & 1111 & 515 & 160 & 282 & 957 \\
\hline
\end{tabular}

* Les «0» viennent de la différence entre l'estimation et le nombre de personnes qui ont reçu au moins un constat.

Deux raisons viennent appuyer la validité de l'estimation de la population à risque. Premièrement, les estimations présentées au tableau 4, basées sur la formule de Zelterman, sont cohérentes avec les estimations

16. Il s'agit de la population itinérante à risque de recevoir un constat pour n'importe quelle infraction non criminelle. Les données disponibles ne distinguent pas les infractions commises, de sorte qu'il n'est pas possible d'estimer les populations en fonction de leur «spécialisation». Par exemple, certaines personnes itinérantes peuvent consommer des boissons alcooliques, mais ne jamais uriner sur la place publique; ainsi, ces personnes ne sont pas à risque de recevoir un constat pour cette infraction. Il est donc envisageable que nos estimations sous-estiment les populations réellement à risque. Toutefois, une analyse des motifs des constats d'infraction remis aux personnes les plus «judiciarisées» (10 constats ou plus en 2009) révèle que la plupart des personnes itinérantes reçoivent des constats pour une variété d'infractions, ce qui suggère que les infractions reprochées sont interchangeables, c'est-à-dire que le motif a relativement peu d'importance. 
générées à l'aide de la formule de Chao (1987) (dans l'ordre, 3257 , $3221,2880,1950$ et 1786 personnes itinérantes) ${ }^{17}$. L'estimateur de Chao génère presque toujours des estimations plus conservatrices que celui de Zelterman (Wilson et Collins, 1992). Deuxièmement, les populations des cinq années ont été estimées de façon indépendante et présentent relativement peu de variation d'une année à l'autre. Toutefois, 2007 est une année charnière : la taille de la population à risque a alors diminué de plus de $30 \%$ par rapport à l'année précédente. Cette diminution coïncide avec l'implantation de l'unité métro, mais est probablement le résultat d'une consigne explicite de réduire le recours aux constats d'infraction pour les personnes itinérantes qui fréquentaient le métro. Cette consigne semble avoir particulièrement touché les personnes qui recevaient un seul constat $(-23 \%)$, puisque le nombre de personnes qui ont reçu deux constats ou plus a légèrement augmenté $(+4 \%)$ de 2007 à 2008. Il y a donc eu, au cours des années 2005 à 2009, un resserrement de la population itinérante à risque de recevoir un constat d'infraction à Montréal.

Les estimations suggèrent que le nombre de personnes itinérantes à risque de recevoir un constat d'infraction est inférieur au nombre de personnes itinérantes présentes à Montréal. En supposant une population d'environ 14000 personnes, la «judiciarisation» toucherait de $13 \%$ à $27 \%$ des personnes itinérantes, selon les années; la proportion diminue si on considère une population plus élevée. Ces résultats n'appuient pas l'hypothèse de la criminalisation, puisque de $73 \%$ à $87 \%$ des personnes itinérantes ne courent pas le risque de recevoir un constat d'infraction. Toutefois, les risques sont élevés pour les autres: de $41 \%$ à $53 \%$ de ces personnes reçoivent au moins un constat d'infraction. Il s'agit de risques considérables, surtout lorsqu'on les compare aux risques d'arrestation estimés pour d'autres infractions.

\section{Discussion et conclusion}

L'analyse présentée n'appuie pas l'hypothèse de la criminalisation de l'ensemble des personnes qui vivent en situation d'itinérance. Toutefois, elle traite d'un seul aspect du problème, la surreprésentation des per-

17. L'estimateur $\mathrm{Z}$ de Zelterman est donné par $\mathrm{N}=\mathrm{n} /\left(1-\mathrm{e}^{\left(-2^{*} \mathrm{n} 2 / \mathrm{n} 1\right)}\right)$, tandis que celui de Chao est donné par $\mathrm{N}=\mathrm{n}+\left(\mathrm{n} 1^{2} / 2^{\star} \mathrm{n} 2\right)$, où $n$ est le nombre d'individus qui ont reçu au moins un constat d'infraction, $n 1$ est le nombre d'individus qui ont reçu un seul constat et $n 2$, le nombre d'individus qui en ont reçu deux. 
sonnes itinérantes parmi les individus qui reçoivent des constats d'infraction. Il serait pertinent de s'intéresser au traitement judiciaire des constats d'infraction remis à des personnes itinérantes, puisque la police ne fait ainsi qu'enclencher le processus de criminalisation. De plus, l'analyse ne tient pas compte du fait que les constats d'infraction n'ont pas le même impact selon la personne qui les reçoit. Enfin, la généralisation des résultats reste à démontrer, puisqu'elle porte sur la situation d'une seule ville et d'un seul service de police au cours d'une période relativement courte (5 ans).

Peut-être de façon plus importante, tous les résultats obtenus dépendent de notre capacité à distinguer les constats d'infraction émis à des personnes itinérantes. La méthode utilisée suppose qu'à défaut d'adresse de domicile fixe, la majorité des personnes itinérantes indiqueront l'adresse d'une des ressources communautaires de Montréal. Il s'agit sûrement d'une sous-estimation, axée principalement sur les personnes itinérantes qui utilisent les ressources communautaires. À défaut de données plus précises sur la condition d'habitation des individus, la méthodologie employée offre au moins une base comparative (voir aussi Bellot et al., 2005). Toutefois, nous avons validé la méthode auprès de policiers, qui nous ont confirmé qu'ils indiquaient l'adresse de ressources dans la majorité des cas.

Les résultats sont cohérents, puisqu'ils vont tous dans la même direction. Les constats émis à des personnes itinérantes ressemblent beaucoup à ceux émis à des personnes non itinérantes, sur le plan du type d'infraction reprochée, du montant de l'amende exigée et du motif de l'intervention (une proportion considérable découle directement de la plainte d'un citoyen). Les circonstances dans lesquelles les personnes reçoivent les constats semblent toutefois varier. L'analyse ne visait pas à déterminer si l'émission de constats d'infraction est une «bonne» stratégie, mais plutôt à documenter la situation montréalaise.

Il est évident que les personnes itinérantes reçoivent une proportion anormalement élevée des constats d'infraction non criminelle, particulièrement au centre-ville et dans le métro. Les sanctions pénales ne sont toutefois pas généralisées à l'ensemble de la population itinérante. Les personnes qui reçoivent un ou plusieurs constats ont des caractéristiques sociodémographiques différentes des utilisateurs de ressources communautaires recensés par Fournier et al. (1998). Ainsi, en retenant l'estimation de 14000 personnes itinérantes à Montréal, de $73 \%$ à $87 \%$ des personnes itinérantes ne risquent pas de recevoir un constat d'infraction, 
dans la situation actuelle. Parmi les personnes à risque, environ la moitié ont reçu au moins un constat, en 2008-2009. Ces personnes se distinguent vraisemblablement quant à la sévérité de leurs problèmes et des endroits qu'elles fréquentent. Autrement dit, l'analyse systématique des constats d'infraction aux règlements municipaux ne permet pas de conclure que la judiciarisation est un phénomène étendu à l'ensemble des personnes itinérantes de Montréal. Les études qui vont dans ce sens semblent souffrir de biais liés à la généralisation de leurs résultats, puisqu'elles tirent leurs conclusions de cas qui ne sont pas représentatifs de la réalité.

Si l'hypothèse de la criminalisation générale de la population itinérante à Montréal n'est pas démontrée, il demeure que de 2000 à 3000 personnes itinérantes courent un risque élevé (environ $50 \%$ ) de recevoir au moins un constat d'infraction au cours d'une année. À qui en revient la responsabilité? À la personne qui commet une infraction? Au citoyen qui porte plainte? Au policier qui décide d'émettre le constat? La responsabilité individuelle des acteurs apparaît limitée, du point de vue de la genèse de l'itinérance (voir aussi Charest, 2003). Plusieurs auteurs ont démontré qu'un grand nombre de facteurs pouvaient contribuer à l'itinérance d'une personne, dont la désinstitutionnalisation des personnes qui souffrent de maladie mentale (Morabito, 2007) et la pénurie de services spécialisés (Elliott et Krivo, 1991; Markowitz, 2006). Nos résultats indiquent aussi que les patrouilleurs ne sont pas appelés à intervenir auprès de toutes les personnes itinérantes. Nombreuses sont celles qui ne seront jamais interpellées par la police. Cela dit, les raisons pour lesquelles les patrouilleurs interviennent auprès de personnes itinérantes sont souvent associées à leurs conditions de vie très précaires et aux problématiques avec lesquelles elles sont aux prises.

Dans ce contexte, ne serait-il pas plus pertinent de demander: «Pourquoi incombe-t-il à la police de gérer les problèmes manifestes des personnes itinérantes?» Face à des plaintes et des désordres bien réels, les policiers doivent intervenir à l'aide des outils d'intervention qui leur sont disponibles. Ceux-ci seront toujours limités par la nature de leur mission de protection du public. Il apparaît ainsi essentiel que toutes les instances concernées par le phénomène de l'itinérance travaillent de concert de manière à avoir une réponse adaptée tant aux besoins des personnes itinérantes qu'aux demandes et préoccupations des citoyens. 


\section{Références}

Bellot, C., Raffestin, I., Royer, M.-N., \& Noël, V. (2005). Judiciarisation et criminalisation des populations itinérantes à Montréal. Montréal: Secrétariat national des sans-abris.

Berk, R., \& MacDonald, J. (2010). Policing the homeless: An evaluation of efforts to reduce homeless-related crime. Criminology and public policy, 9 (4), 813-840.

Berry, B. (2007). A repeated observation approach for estimating the street homeless population. Evaluation review, 31 (2), 166-199.

Bouchard, M. (2007). A capture-recapture model to estimate the size of criminal populations and the risks of detection in a marijuana cultivation industry. Journal of quantitative criminology, 23, 221-241.

Bouchard, M., \& Tremblay, P. (2005). Risks of arrest across markets : a capturerecapture analysis of 'hidden' dealer and user populations. Journal of drug issues, 34, 733-754.

Brassard, R., \& Cousineau, M.-M. (2002). Les coups de l'itinérance: une étude sur la victimisation criminelle du point de vue des itinérants. Les cahiers de recherche criminologique (cahier no 34). Montréal: Centre international de criminologie comparée, Université de Montréal.

Chao, A. (1987). Estimating the population size for capture-recapture data with unequal catchability. Biometrics, 43, 783-791.

Charest, M. (2010). L'évolution des populations de trafiquants et de voleurs à Montréal, 1997-2008. In M. Côté (ed.), Lecture de l'environnement du Service de police de la ville de Montréal (102-113). Montréal: SPVM, Direction stratégique, Section de la recherche et de la planification.

Charest, R. (2003). Police et prise en charge des personnes itinérantes à Montréal. Politiques sociales, 1-2, 66-77.

Collins, M. F., \& Wilson, R. M. (1990). Automobile thefts: Estimating the size of the criminal population. Journal of quantitative criminology, 6, 395-409.

Elliott, M., \& Krivo, L. J. (1991). Structural determinants of homelessness in the United States. Social problems, 38 (1), 113-131.

Engel, R. S., \& Silver, E. (2001). Policing mentally disordered suspects: A reexamination of the criminalization hypothesis. Criminology, 39 (2), 225-252.

Fazel, S., Khosla, V., Doll, H., \& Geddes, J. (2008). The prevalence of mental disorders among the homeless in western countries: Systematic review and meta-regression analysis. PLOS Medicine, 5 (12), 1670-1681.

Fisher, N., Turner, S. W., Pugh, R., \& Taylor, C. (1994). Estimating numbers of homeless and homeless mentally ill people in north east Westminster by using capture-recapture analysis. British Medical Journal, January, 27-30.

Fitzpatrick, K. M., La Gory, M. E., \& Ritchey, F. J. (1993). Criminal victimization among the homeless. Justice quarterly, 10 (3), 353-368.

Fournier, L. (2001). Enquête auprès de la clientèle des ressources pour personnes itinérantes des régions de Montréal-Centre et de Québec, 1998-1999. Québec: Institut de la statistique du Québec. 
Fournier, L., Chevalier, S., Ostoj, M., Caulet, M., Courtemanche, R, \& Plante, N. (1998). Dénombrement de la clientèle itinérante dans les centres d'bébergement, les soupes populaires et les centres de jour des villes de Montréal et de Québec, 19961997. Québec, QC: Santé Québec.

Gaetz, S. (2006). Safe streets for whom? Homeless youth, social exclusion and criminal victimization. Canadian journal of criminology and criminal justice/La revue canadienne de criminologie et de justice pénale, 46 (4), 423-456.

Greenberg, G. A., \& Rosenheck, R. A. (2008). Jail incarceration, homelessness, and mental health: A national study. Psychiatric services, 59 (2), 170-177.

Guthrie, J. (2010, 12 juillet) Plus d'itinérants en raison de la pénurie de logements. Le journal Métro.

Institut canadien d'information sur la santé (ICIS) (2007). Améliorer la santé des Canadiens, 2007-2008: santé mentale et itinérance. Ottawa: ICIS.

Landreville, P., Laberge, D., \& Morin, D. (1998). La criminalisation et l'incarcération des personnes itinérantes. Nouvelles pratiques sociales, 11 (1), 69-81.

Larouche, A. (2009). L'expérience carcérale dans le parcours des jeunes de la rue à Montréal. Champ pénal, VI. Consulté le 9 juillet 2010, < http:// champpenal.revues.org $>$.

Lee, B. A., \& Schreck, C. J. (2005). Danger on the streets: Marginality and victimization among the homeless people. American behavioural scientist, 48 (8), 1055-1081.

Markowitz, F. E. (2006). Psychiatric hospital capacity, homelessness, and crime and arrest rates. Criminology, 44 (1), 45-72.

Markowitz, F. E., Bellair, P. E., Liska, A. E., \& Liu, J. (2001). Extending social disorganization theory: Modeling the relationships between cohesion, disorder and fear. Criminology, 39, 293-320.

McCarthy, B., \& Hagan, J. (1991). Homelessness: A criminogenic situation? British journal of criminology, 31 (4), 393-410.

McGuire, J. F., \& Rosenheck, R. A. (2004). Criminal history as a prognostic indicator in the treatment of homeless people with severe mental illness. Psychiatric services, 55 (1), 42-48.

Metraux, S., \& Culhane, D. P. (2006). Recent incarceration history among a sheltered homeless population. Crime $\mathcal{O}$ Delinquency, 52 (3), 504-517.

Morabito, M. S. (2007). Horizons of context: Understanding the police decision to arrest people with mental illness. Psychiatric services, 58 (12), 1582-1587.

National Law Center on Homelessness and Poverty (NLCHP). (2009). Homes not handcuffs: The criminalization of homelessness in $U$. S. cities. Washington D. C. : NLCHP.

Newcombe, R. G. (1998). Interval estimation for the difference between independent proportions: Comparison of eleven methods. Statistics in Medicine, 17 (8), 873-890.

Novak, K. J., \& Engel, R. S. (2005). Disentangling the influence of suspects' demeanor and mental disorder on arrest. Policing, 28 (3), 493-512.

Pogrebin, M. R. (1986). Police responses for mental health assistance. Psychiatric quarterly, $58(1)$, 66-73. 
Radio-Canada. (5 juillet 2010). Les refuges de Montréal débordés. Consulté le 12 juillet 2010, <Radio-Canada.ca $>$.

Raffestin, I. (2009). Une injustice programmée? Le point de vue des personnes itinérantes sur leur judiciarisation et leur incarcération (Mémoire de maîtrise). École de service social, Université de Montréal, Montréal.

Roberts, J. M., \& Brewer, D. D. (2006). Estimating the prevalence of male clients of prostitute women in Vancouver with a simple capture-recapture method. Journal of the Royal statistical society: Series A, 169 (4), 745-756.

Robitaille, C., Guay, J.-P., \& Savard, C. (2002). Portrait de la clientèle correctionnelle du Québec 2001. Montréal: Société de criminologie du Québec pour la DGSC du ministère de la Sécurité publique du Québec.

Rossi, P. H., Wright, J. D., Fisher, G. A., \& Willis, G. (1987). The urban homeless: Estimating composition and size. Science, 235 (4794), 1336-1341.

Rossmo, D. K., \& Routledge, R. (1990). Estimating the size of criminal populations. Journal of quantitative criminology, 6, 293-314.

Roy, E., Haley, N., Leclerc, P., Cedras, L., Blais, L., \& Boivin, J.-F. (2003). Drug injection among street youths in Montreal: Predictors of initiation. Journal of Urban Health, 80 (1), 92-105.

Roy, S., \& Hurtubise, R. (2007). L'itinérance en questions. Sainte-Foy: Presses de 1'Université du Québec.

Roy, S., \& Morin, D. (2007). L'aide aux personnes itinérantes: un réseau pour agir. In S. Roy \& R. Hurtubise (eds.), L'itinérance en questions (195-215). Sainte-Foy: Presses de l'Université du Québec.

Skogan, W. G. (1990). Disorder and decline: crime and the spiral decay of American neighborboods. New York: Free Press.

Snow, D. A., Baker, S. G., \& Anderson, L. (1989). Criminality and homeless men: An empirical assessment. Social problems, 36 (5), 532-549.

Société de transport de Montréal (2008). La STM en 2008. Rapport d'activités. Consulté le 28 novembre 2012, www.stm.info/en-bref/vu2008.pdf.

Sylvestre, M.-E. (2008). La pénalisation et la judiciarisation des personnes itinérantes au Québec: des pratiques coûteuses, inefficaces et contre-productives dans la prévention de l'itinérance et la réinsertion des personnes itinérantes. Mémoire remis à la commission parlementaire sur l'itinérance, Québec.

Sylvestre, M.-E. (2010). Disorder and public spaces in Montreal: Repression (and resistance) through law, politics, and police discretion. Urban Geography, 31 (6), 803-824.

Teplin, L. A. (1984). Criminalizing mental disorder: The comparative arrest rate of the mentally ill. American Psychologist, 39, 794-803.

van Hest, N. A. H., De Vries, G., Smit, F., Grant, A. D., \& Richardus, J. H. (2008). Estimating the coverage of a targeted mobile tuberculosis screening programme among illicit drug users and homeless persons with truncated models. Epidemiology and Infection, 136, 628-635.

Wilson, R. M., \& Collins, M. F. (1992). Capture-recapture estimation with samples of size one using frequency data. Biometrika, 79, 543-553. 
Wilson, J. Q., \& Kelling, G. L. (1982). Broken windows: Police and neighbourhood safety. Atlantic Monthly, 249, 29-38.

Zelterman, D. (1988). Robust estimation in truncated discrete distributions with application to capture-recapture experiments. Journal of statistical planning and inference, 18 (2), 225-237.

\begin{abstract}
Police interventions towards homeless individuals are frequently criticized, especially when it involves issuing statements of offence. This article explores one aspect of the criminalization hypothesis by analyzing data on statements of non criminal offences issued in Montreal, between 2005 and 2009. Our results indicate that statements handed over to homeless individuals are not significantly different from those issued to non-homeless. However, homeless individuals receive on average three times more statements, which suggest that police intervene differently when dealing with homeless people. Nevertheless, not all homeless individuals run the risk of receiving a statement of offence. Our results put into perspective the alarmist position frequently presented by mass media.
\end{abstract}

KEYWORDS • Homelessness, police, police intervention, criminalization.

RESUMEN - Las intervenciones policiacas contra personas que viven en la calle son con frecuencia objeto de protestas en la plaza pública, en particular cuando incluyen multas por infracción a reglamentos municipales. El presente artículo analiza la representación desproporcionada de las poblaciones itinerantes de Montreal entre las personas que recibieron al menos una boleta de multa en el periodo 2005-2009. Los resultados indican que los itinerantes reciben un número promedio de multas tres veces más grande. Sin embargo, las tres cuartas partes de los itinerantes no corren ningún riesgo de recibir una boleta de infracción en el curso del año. Los resultados obtenidos ponen en perspectiva la visión alarmista que con frecuencia se transmite en los medios de comunicación.

PALABRAS CLAVE - Itinerantes, policía, intervención policiaca, reglamentos municipales. 
ANNEXE 1

Nombre de constats d'infraction par mois, personnes itinérantes et non itinérantes, janvier 2005-décembre 2009

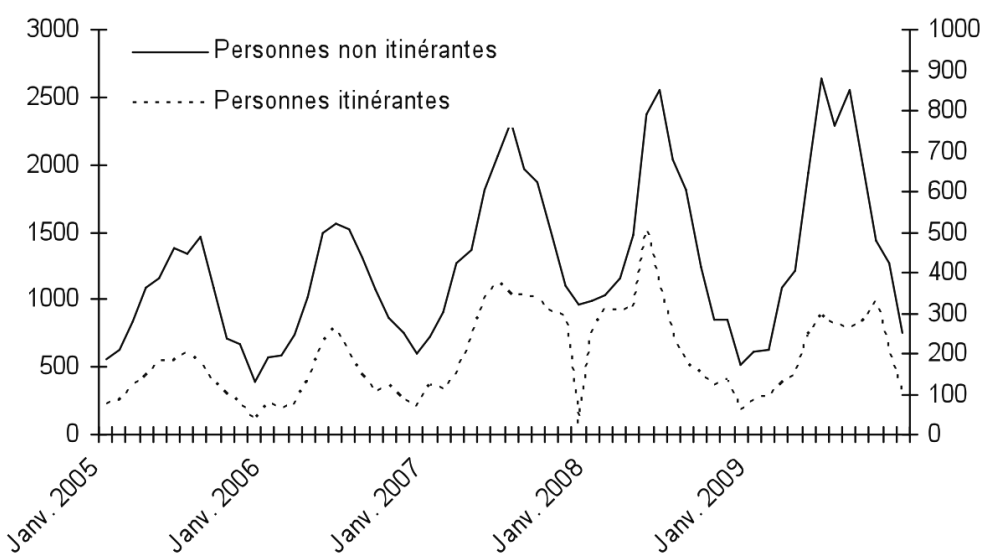

Note: Chaque mois, un plus grand nombre de constats d'infraction sont remis à des personnes non itinérantes qu'à des personnes itinérantes. Pour faciliter la visualisation, les courbes ont été rapprochées. Ainsi, l'échelle de gauche correspond au nombre de constats remis à des personnes non itinérantes, tandis que l'échelle de droite correspond au nombre de constat remis à des personnes itinérantes. 